\title{
Apoyo social, resiliencia y región de origen en la salud mental y la satisfacción vital de los inmigrantes
}

Social Support, Resilience and Place of Origin in Immigrants' Mental Health and Vital Satisfaction

Recepción: 23 Mayo 2017 | Aprobación: 28 Agosto 2017

\author{
Alba García-Cid \\ Universidad de Málaga, España \\ IsABel Hombrados-Mendieta ${ }^{\mathrm{a}}$ \\ Universidad de Málaga, España \\ ORCID: http://orcid.org/0000-0001-7288-1589 \\ Luis Gómez-Jacinto \\ Universidad de Málaga, España \\ María de las Olas Palma-García \\ Universidad de Málaga, España \\ Mario Millán-Franco \\ Universidad de Málaga, España
}

a Autor de correspondencia. Correo electrónico: mihombrados@uma.es

Para citar este artículo: García-Cid, A., HombradosMendieta, I., Gómez-Jacinto, L., Palma-García, M O., \& Millán-Franco, M. (2017). Apoyo social, resiliencia y región de origen en la salud mental y la satisfacción vital de los inmigrantes. Universitas Psychologica, 16(5), 1-14. https://doi.org/10.11144/Ja veriana.upsy16-5.asrr

\section{RESUMEN}

Se analiza la relación del apoyo social y la resiliencia con la satisfacción vital y la salud mental de los inmigrantes, y las diferencias en dichas variables según la región de origen (África, Europa, Latinoamérica y Asia). En el estudio han participado 1094 inmigrantes que residen en España. Los resultados de la regresión por pasos muestran que la tenacidad, el apoyo social de la familia y de los autóctonos, y el control personal son los mejores predictores de la satisfacción vital. La tenacidad, el apoyo de la familia y el control lo son de la salud mental.

\section{Palabras clave}

inmigrantes; apoyo social; resiliencia; satisfacción vital; salud mental.

\section{ABSTRACT}

We analyze the relationship between social support and resilience with vital satisfaction and mental health in immigrants, and if there are differences in these variables according to the immigrants' place of origin (Africa, Europe, Latin America and Asia). 1094 immigrant subjects who live in Spain have taken part. The stepwise regression results show that tenacity, social support from family and immigrant friends, and personal control are the best predictors of the vital satisfaction. Tenacity, social support from family and personal control are the best predictors of the mental health.

Keywords

immigrants; social support; resilience; life satisfaction; mental health. 
Las migraciones han tenido diferentes formas a lo largo de la historia, y han compuesto una realidad con características propias, donde se encuentran cada vez más países y más religiones, lo que complica el desplazamiento y visibiliza a diferentes grupos migratorios, con motivaciones y singularidades propias (Pereda \& Pereira, 2006). Muchos son los estresores a los que este colectivo debe hacer frente. Algunos de ellos serían el lenguaje, la barrera de la comunicación, las dificultades económicas, el cambio sociocultural, la falta de trabajo, la exclusión social o la pérdida del apoyo familiar y social (Zarza \& Prados, 2007), además de la discriminación y amenazas que ponen en riesgo su integridad física (UgaldeWatson, Smith-Castro, Moreno-Salas, \& Rodríguez-García, 2011); factores que afectan su satisfacción vital y salud mental. Los estudios sobre integración y bienestar de los inmigrantes han prestado especial atención a los indicadores subjetivos del bienestar y a profundizar acerca de cómo se sienten los inmigrantes en la sociedad de acogida.

Hay un área de investigación que sugiere que la interacción social positiva y el apoyo social son variables importantes para explicar el bienestar y la salud (Malone \& Dooley, 2006). Otros estudios se han centrado más en el análisis de las variables personales; el análisis de la resiliencia o capacidad de afrontamiento a la adversidad y la capacidad para desarrollar competencias ante situaciones estresantes es de especial interés (Barudy \& Dantagnan, 2005). Por tanto, más que analizar los elementos que conducen a la exclusión, en el presente estudio nos centraremos en el análisis de las variables psicosociales positivas como el apoyo social y la resiliencia y su relación con la satisfacción vital y la salud mental en los diferentes grupos de inmigrantes.

\section{Apoyo social e inmigración}

Se han comprobado los efectos positivos del apoyo social sobre el bienestar y la salud mental de los inmigrantes. Lin, Dean y Ensel (1986) lo definen como una provisión instrumental y expresiva, ya sea real o percibida, cuyas fuentes podrían ser la familia, los amigos, la comunidad y las redes sociales más extensas. El papel del apoyo social sobre la población inmigrante ha sido avalado por algunos modelos como el Modelo del Convoy Social de Khan y Antonucci (1980) que explica cómo las redes sociales se adaptan en el proceso de transición según las propias características del inmigrante y del contexto. Diversos estudios señalan la importancia de gozar de relaciones y vínculos cercanos para compartir las vivencias y los problemas personales, y de que dichas redes tengan la capacidad de procurar la ayuda requerida, para que así la percepción de apoyo social sea idónea y conveniente (Hovey \& Magana, 2002). En inmigrantes, el apoyo social protege la salud mental, gracias a la convivencia con una red social amplia, el apoyo social emocional y la satisfacción con dichas relaciones (Herrero \& Gracia, 2011). Además, tiene efectos positivos en la salud, en la valoración que se hace de la vida y en el bienestar global (Lai \& McDonald, 1995). Sin embargo, las interacciones negativas entre iguales y familiares perjudican gravemente la salud mental, provocando cuadros depresivos (Xu \& Chi, 2013). Los estudios sobre las fuentes que proporcionan apoyo a la población inmigrante muestran resultados contradictorios. Algunos indican que los inmigrantes establecen un mayor número de relaciones positivas entre ellos, y que las relaciones con los autóctonos son más limitadas y sobre todo de carácter más informacional (Hernández, Alonso, \& Pozo, 2006). En cambio, otros estudios señalan los efectos beneficiosos sobre el bienestar del apoyo de la familia, de la población autóctona y de las asociaciones (Domínguez \& Hombrados, 2012).

En cuanto a la percepción de apoyo según el lugar de origen de los inmigrantes, los estudios muestran que el impacto del apoyo social es distinto en los diferentes grupos étnicos y culturales, en donde los latinoamericanos son los que en general perciben más apoyo frente a otros grupos de inmigrantes (JasinskajaLahti, Liebkind, Jaakkola, \& Reuter, 2006) y sufren menos las consecuencias del estrés por aculturación sobre la salud que los asiáticos (Panchang, Dowdy, Kimbro, \& Gorman, 
2016). Algunos estudios apuntan a que los latinoamericanos perciben más apoyo porque pertenecen a culturas colectivistas-horizontales, donde se promueve la sociabilidad entre iguales frente a otras culturas colectivistasverticales como la asiática, donde los procesos de interacción social se producen de forma jerárquica, o las culturas individualistas como la africana, que suelen mostrar valores más bajos de apoyo social (Shavitt et al., 2016). También se ha encontrado que los asiáticos utilizan menos las redes de apoyo para afrontar el estrés que los europeos (Wang \& Lang, 2015).

\section{Resiliencia e inmigración}

Otra variable que contribuye a afrontar de forma positiva las situaciones adversas es la resiliencia, definida como "La capacidad humana de adaptarse a la tragedia, el trauma, la adversidad, las dificultades y los constantes factores de estrés en la vida" (Newman, 2005, p.1). Se refiere a la capacidad de la persona, de los grupos familiares y/o comunidades para hacer frente a las adversidades con las que se encuentran, a las dificultades personales, sociales y laborales. Representa la habilidad añadida de saber superar dichas adversidades, aprendiendo de ellas y fortaleciéndose desde la experiencia vivida (Luthar, Cicchetti, \& Becker, 2000). Es la relación con la adversidad lo que hace de la resiliencia una variable de interés para ser estudiada en la población inmigrante pues, como apuntan algunos autores, las personas resilientes tienen más capacidad de integrarse en la comunidad (Rutter, 1993), en donde esto es especialmente importante para la población inmigrante. La disposición a estar con otras personas permitiría desarrollar la resiliencia de manera interpersonal (Barudy \& Dantagnan, 2005). Por tanto, se hace evidente la necesidad de estudio de dicha capacidad en la inmigración, sobre todo si atendemos a que en la mayoría de investigaciones el proceso migratorio es tratado como un proceso traumático (Obrist \& Büchi, 2008).
Se destacan tres elementos importantes de la resiliencia: los recursos psicológicos y de afrontamiento, como la autoestima, el optimismo y la autoeficacia; el apoyo social de la familia, los amigos o asociaciones, y el entorno comunitario como los servicios de salud, servicios sociales, etc. (Gagnon \& Stewart, 2014). Entre los elementos que componen la resiliencia, se destacan la autoeficacia, la sensación de control y la competencia social, ya que constituyen los rasgos esenciales de las personas consideradas resilientes (Connor \& Davidson, 2003). Para Serrano-Parra et al. (2012), estas dimensiones son las que mejor definen la resiliencia, pues se considera la dimensión de tenacidad-autoeficacia como un elemento mediador en los procesos de recuperación tras diversos tipos de daño, que puede ayudar a mantener la seguridad ante situaciones adversas, permitiendo una mejor adaptación a los desafíos vitales. La dimensión de control personal hace referencia a poseer tanto objetivos claros como una alta percepción de autocontrol cognitivo y emocional.

Esta capacidad de autorregulación es un buen predictor de la salud, el autocuidado y la competencia social, que contribuye a modular emociones negativas a través de la interacción con los demás, lo que la convierte en un factor protector frente a los estímulos estresores. Además, favorece la conexión con las redes sociales de apoyo, permite optimizar los recursos y generar un sistema de resiliencia personal, familiar y comunitario.

La resiliencia se ha considerado como un factor protector de trastornos depresivos, ansiógenos y de estrés postraumático (Herrman et al., 2011), y se ha asociado a una buena calidad de vida y satisfacción vital (Luthar \& Cichetti, 2000; Mahendra \& Agarwal, 2016). También se ha observado que el apoyo social dota a la persona inmigrante de resiliencia, lo que disminuye las probabilidades de depresión y reduce el riesgo de ingreso clínico (Kiang, Grzywacz, Marín, Arcury, \& Quandt, 2010). También se han hallado diferencias en cuanto al lugar de origen y la resiliencia desarrollada en el proceso de inmigración: las personas procedentes de Sudamérica son las que desarrollan más 
resiliencia, seguidas de europeos y africanos (Vélez, Aguado, \& Pérez, 2013).

\section{Salud mental e inmigración}

Existe cierto consenso entre los autores en relacionar las dificultades y eventos estresantes a los que deben hacer frente las personas inmigrantes a lo largo de su vida, con las consecuencias que suponen para su estabilidad y salud mental, y se considera como un suceso de crisis vital (Tunstall, Shortt, Pearce, \& Mitchell, 2015). El nuevo idioma, el alejamiento de sus seres queridos, el riesgo mismo del viaje, la precariedad laboral, pésimas condiciones de vida y pobreza, aislamiento social, etc., provocan estrés, indefensión, ansiedad y depresión (Shoval, Schoen, Vardi, \& Zalsman, 2007). Por ello, los factores de protección y la supresión de factores de riesgo contribuyen a una buena salud mental que estaría positivamente relacionada con el apoyo social y la integración social (Hombrados, Gómez, Domínguez, \& García, 2013; Patiño \& Kirchner, 2008). Algunos estudios también muestran que existen diferencias en salud mental en los diferentes grupos de inmigrantes según su región de origen; aquellos que proceden de países de Europa Occidental valoran su salud de forma más similar a la ciudadanía española, mientras que los procedentes de Marruecos, Ecuador y Europa del Este tienen una percepción más negativa (Tormo et al., 2015). Asimismo, se han encontrado diferencias en integración en el país de acogida en jóvenes según su mayor o menor similitud cultural, esto es, los jóvenes latinoamericanos y de Europa del Este manifiestan una mayor adaptación frente a los jóvenes africanos (Checa \& Monserrat, 2015). Los autores lo explican a partir de la semejanza del idioma y la religión de raíces comunes.

\section{Satisfacción con la vida e inmigración}

La satisfacción con la vida o satisfacción vital se entiende como una evaluación cognitiva que la persona hace de su propia vida, como el bienestar integral o global, utilizando criterios individualmente elegidos y comparándolos con las expectativas y estándares previos de la persona (Diener, 2006).

En general, se ha prestado más atención a los elementos de riesgo y desadaptación que a aquellos que preservan la calidad de vida (Rudmin, 2009), en donde la aproximación a la cultura de acogida (Ayyash-Abdo \& Alamuddin, 2007) y el apoyo social son algunos factores que pueden predecir una buena satisfacción vital (Domínguez \& Hombrados, 2012). Atendiendo a las relaciones interpersonales, el grado de comunicación y la afinidad con el país receptor predicen la satisfacción vital (Safi, 2010). La integración social en la comunidad cumple un importante papel en la satisfacción vital (Herrero, Gracia, Fuente \& Lila, 2012). También se han encontrado diferencias en satisfacción vital en los diferentes grupos de inmigrantes según la aproximación a la cultura de acogida, en donde los magrebíes y subsaharianos son los menos satisfechos, comparados con la población latina y de Europa del Este (Moreno \& Hidalgo, 2011).

\section{Estudio actual}

El fenómeno migratorio implica numerosas situaciones de vulnerabilidad que afectan a la satisfacción vital y a la salud mental de la población inmigrante. En dicho proceso, la percepción de apoyo social y la resiliencia pueden contribuir a reducir los efectos negativos. Este estudio se plantea como objetivos analizar la relación entre el apoyo social, la resiliencia, la satisfacción vital y la salud mental en la población inmigrante, y comprobar si existen diferencias en los diferentes grupos de inmigrantes según la región de origen (África, Asia, Latinoamérica y Europa del Este) en las variables analizadas. En la Figura 1 se muestra la red de relaciones teóricas que se pretende poner a prueba. 


\section{Figura 1}

Diagrama de las relaciones teóricas entre las variables del estudio

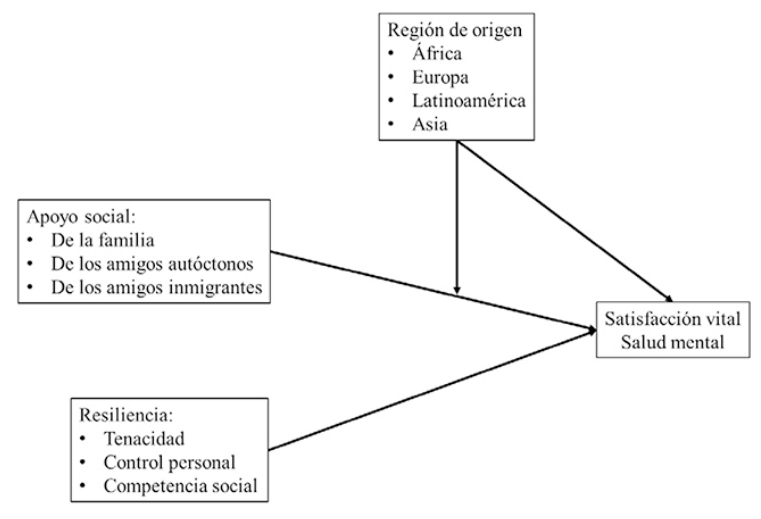

Fuente: elaboración propia.

Hemos definido como hipótesis:

Hipótesis 1: la región de origen de los inmigrantes predice su satisfacción con la vida y sus problemas mentales, teniendo en cuenta que las de mayor cercanía cultural con la sociedad de acogida (Europa del Este y Latinoamérica) manifestarán mayor satisfacción con la vida y menor incidencia de los problemas mentales que las de menor cercanía cultural con la sociedad de acogida (África, Asia).

Hipótesis 2: las dimensiones de la resiliencia (tenacidad-autoeficacia, control personal y competencia social), el apoyo social proporcionado por la familia, los amigos autóctonos y los amigos inmigrantes se relacionan positivamente con la satisfacción con la vida y con una menor incidencia de los problemas mentales.

Hipótesis 3: la región de origen modula la influencia del apoyo social recibido y la resiliencia sobre la satisfacción vital y los problemas mentales de la población inmigrante.

\section{Método}

\section{Participantes}

En el estudio participaron 1094 inmigrantes, de los cuales un $52.9 \%$ son mujeres (579) y un $47.1 \%$ son hombres (515). La edad osciló entre los 16 y $\operatorname{los} 80$ años, con una media de 32.26 años.
En cuanto a la procedencia, el $27.4 \%$ son de Latinoamérica, el 24.7\% de Asia, el 24.4\% de África y el 23.7\% de Europa del Este. Esto es representativo de la distribución de inmigrantes en la ciudad según los datos del censo (2015). Los participantes llevan una media de 10.27 años fuera de su país de origen y el tiempo medio de residencia en Málaga es de 8.71. La mayoría de los inmigrantes son solteros (49\%), están empleados el 53.3\%, el motivo principal de abandono del país es mejorar la economía (49.2\%). En cuanto al nivel de estudios el 24\% tiene estudios secundarios terminados y solo el $12 \%$ estudios universitarios acabados.

\section{Procedimiento}

El estudio se realiza en Málaga (España), ciudad que se divide administrativamente en once distritos municipales que se tomaron como referencia para ubicar y seleccionar los núcleos que podían resultar de mayor interés para el estudio. De todos los posibles barrios que componen cada distrito, se han seleccionado aquellos en los que hay mayor presencia de inmigrantes.

Dada la dificultad de contactar con la población inmigrante, los participantes se seleccionaron en los lugares donde era más fácil su localización, como asociaciones, locutorios ubicados en los barrios, lugares de reunión reconocidos en la ciudad, lugar de residencia y centros de servicios sociales. Se delimitaron geográficamente en cada uno de los distritos de la ciudad los barrios seleccionados donde se evaluarían a los inmigrantes, y estos se eligieron al azar, utilizando un sistema de rutas, muy indicado en este tipo de investigación. En cada barrio se seleccionaron al azar, sobre un mapa, las rutas correspondientes, indicando las calles, edificios, plantas, viviendas y giros. Dentro de cada distrito se calculó una muestra representativa teniendo en cuenta los datos del censo de la ciudad, por lo que se realizó un muestreo por cuotas en donde quedaba representado el sexo y la procedencia. Los inmigrantes entrevistados no recibieron compensación económica pero sí 
se les proporcionaba datos de contacto para informarles de los resultados del estudio.

\section{Instrumentos}

Características sociodemográficas: se recogieron datos sobre el país de origen, la edad, el sexo, el estado civil, el tiempo de residencia en Málaga, el tiempo de residencia fuera de su país de origen, el motivo de abandono de su país de origen, el nivel de estudios y la situación laboral.

Problemas mentales: se utilizó el General Health Questionnaire de Goldberg GHQ-12, en su versión española (Villa, Zuluaga, \& Restrepo, 2013). El cuestionario está formado por 12 ítems, que son contestados a través de una escala tipo Likert de cuatro puntos (0-3). En la tabla 1 aparece sus estadísticos descriptivos y su valor $\alpha$.

Satisfacción con la vida: escala de Satisfacción con la Vida (Satisfaction With Life Scale, SWLS) de Pavot y Diener (1993) evalúa la satisfacción con la vida o componente cognitivo del bienestar con cinco ítems (e.g. Hasta ahora, he conseguido las cosas que para mí son importantes en la vida). El formato de respuesta es de tipo Likert con 7 opciones de respuesta que van desde $1=$ completamente insatisfecho hasta $7=$ completamente satisfecho. En la tabla 1 aparece sus estadísticos descriptivos y su valor $\alpha$.

Apoyo social: cuestionario de Frecuencia y Satisfacción con el apoyo social (García, Hombrados-Mendieta, \& Gómez, 2016). Mide la frecuencia y satisfacción con el apoyo emocional, instrumental e informacional recibido de la familia, los amigos inmigrantes y los amigos autóctonos. Consta de 18 ítems (e.g. Su familia le da cariño, afecto y le escucha cuando quiere hablar y expresar sus sentimientos). La frecuencia de apoyo se mide a través de una escala Likert con cinco alternativas de respuesta $(1=$ "Rara vez", $5=$ "Siempre"). El grado de satisfacción con el apoyo recibido se mide también con una escala Likert de cinco alternativas ( $1=$ "Insatisfecho", $5=$ "Muy satisfecho"). El apoyo social de cada una de las fuentes es un índice que se construye a partir de la frecuencia y la satisfacción de los tres tipos de apoyo (emocional, instrumental e informacional). En la tabla 1 aparecen sus estadísticos descriptivos y su valor $\alpha$.

Resiliencia: Se utilizó la Escala CD-RISC de Resiliencia en su versión en castellano elaborada por Serrano-Parra et al. (2012). La escala CD-RISC consta de 17 ítems distribuidos en tres dimensiones tenacidad-autoeficacia (e.g. Los éxitos del pasado me dan confianza para enfrentarme con nuevos retos y dificultades); control personal (e.g. Puedo enfrentarme a cualquier cosa que se me presente); y competencia social (e.g. Tengo al menos una relación íntima y segura que me ayuda cuando estoy angustiado). Se responde conforme a una escala tipo Likert desde $1=$ totalmente en desacuerdo a $5=$ totalmente de acuerdo. El alpha de Cronbach de las tres dimensiones se muestra en la Tabla 1.

En la Tabla 1 se presenta un resumen de las variables empleadas en el estudio y la matriz de intercorrelaciones de las mismas.

\section{TABLA 1}

Estadísticos descriptivos y valores $\alpha$ de las variables analizadas

\begin{tabular}{|c|c|c|c|c|c|c|c|c|c|c|c|c|}
\hline & $n$ & Rango & $M$ & $D T$ & $\alpha$ & 1 & 2 & 3 & 4 & 5 & 6 & 7 \\
\hline 1 Tenacidad & 1117 & $1-5$ & 3.88 & 0.62 & 0.79 & & & & & & & \\
\hline 2 Conirol personal & 1115 & $1-5$ & 3.66 & 0.68 & 0.79 & 0.71 & & & & & & \\
\hline 3 Competencia social & 1116 & $1-5$ & 3.80 & 0.82 & 0.56 & 0.60 & 0.59 & & & & & \\
\hline $\begin{array}{l}\text { 4Apoyo de a amumla } \\
5 \text { Apoyo de bs amizos aub́conos }\end{array}$ & $\begin{array}{l}1022 \\
1095\end{array}$ & $\begin{array}{l}1-5 \\
1-5\end{array}$ & $\begin{array}{l}4.05 \\
3.58\end{array}$ & 1.06 & $\begin{array}{l}0.91 \\
0.90\end{array}$ & $\begin{array}{l}0.39 \\
0.32\end{array}$ & $\begin{array}{l}0.28 \\
0.26\end{array}$ & $\begin{array}{l}0.24 \\
0.22\end{array}$ & 0.45 & & & \\
\hline 6 Apoyo de bs amigos irmigrartes & 1081 & $1-5$ & 3.49 & 1.11 & 0.87 & 0.21 & 0.12 & 0.12 & 0.37 & 0.37 & & \\
\hline 7 Satiffacción con h vida & 1113 & $1-7$ & 4.42 & 1.24 & 0.88 & 0.49 & 0.41 & 0.33 & 0.38 & 0.36 & 0.21 & \\
\hline 8 Problemms mentales & 1070 & $0-2.75$ & 0.95 & 0.43 & 0.87 & -0.27 & -0.28 & -0.18 & -0.26 & -0.16 & -0.08 & -0.33 \\
\hline
\end{tabular}

Fuente: elaboración propia.

\section{Resultados}

Para probar las hipótesis uno y dos, se realiza un análisis de regresión múltiple en el que la región de origen, la resiliencia y el apoyo social son las variables predictoras, y la satisfacción con la vida y los problemas mentales, las variables criterio. En la Tabla 2 se muestran los resultados del análisis de regresión múltiple por pasos del criterio satisfacción con la vida. En un primer paso se incluyen en la ecuación las variables ficticias creadas a partir de la variable región de origen del inmigrante. En el paso dos y en los siguientes se van incluyendo el resto de los predictores según su peso. Al final del proceso quedan excluidas, por no ser su influencia estadísticamente significativa, las variables competencia social y apoyo de los 
amigos inmigrantes. En el primer paso se puede observar que hay diferencias entre los cuatro grupos en satisfacción con la vida.

Los latinoamericanos son los que puntúan más alto en esta variable $(M=4.68)$, seguidos por los europeos $(M=4.51)$; algo más lejos están los asiáticos $(M=4.29)$ y los africanos $(M=4.11)$. En el segundo paso, se incrementa fuertemente la proporción de varianza explicada de satisfacción con la vida al entrar en la ecuación la tenacidad. El paso tres también aumenta la $\mathrm{R}^{2}$ con la inclusión en la ecuación del apoyo social proporcionado por los amigos autóctonos. Lo que también sucede en el cuarto paso, con el apoyo social de la familia. Finalmente, entra el control personal. Puede observarse que a partir del modelo 2 desaparecen las diferencias entre los cuatro grupos, salvo para los procedentes de África, que son los que tienen una menor satisfacción con la vida, manteniendo constantes el resto de las variables. Ello implica que la hipótesis 1 no se cumple en líneas generales, pues la diferencia entre los cuatro grupos desaparece cuando se introducen el resto de las variables predictoras; solo se mantiene la diferencia en lo que a los inmigrantes africanos se refiere. Sí se verifica la hipótesis 2 , pues a una mayor tenacidad y control personal, así como a la percepción del apoyo social de los amigos autóctonos y de la familia, le corresponden una mayor satisfacción con la vida, y queda fuera de la predicción el apoyo de los amigos inmigrantes y la competencia social.

Cabe decir que hay una correlación alta $(r$ $=0.71$ ) entre las dos variables de resiliencia que se han retenido en la ecuación de regresión, sin que se hayan resentido excesivamente los diagnósticos de colinealidad. Así, para la variable tenacidad los índices son: Tolerancia $=0.43 ;$ FIV $=2.31$. Para control personal: Tolerancia $=0.48$; $F I V=2.08$. Solo cuando en la ecuación se introduce la variable control personal, aumentan moderadamente los problemas de colinealidad (Autovalor $=0.007$; Índice de condición $=27.57$ ). Ello indica una cierta redundancia de esta variable respecto a la de tenacidad.

\section{TABLA 2}

Regresión múltiple por pasos con satisfacción con la vida como criterio y con región de origen (variables ficticias), resiliencia (tenacidad, control personal y competencia social) y apoyo social (familiar, amigos autóctonos y amigos inmigrantes) como predictores

\begin{tabular}{|c|c|c|c|c|c|c|c|c|c|c|}
\hline & \multicolumn{2}{|c|}{ Paso 1} & \multicolumn{2}{|c|}{ Paso 2} & \multicolumn{2}{|c|}{ Paso 3} & \multicolumn{2}{|c|}{ Paso 4} & \multicolumn{2}{|c|}{ Paso 5} \\
\hline & $\Delta R^{2}=0.0$ & $F=11.13$ & $\Delta R^{2}=0.2$ & $F=91.39$ & $\Delta R^{2}=0.0$ & $F=93.81$ & $\Delta R^{2}=0.0$ & $F=84.95$ & $\Delta R^{2}=0$ & $F=74.90$ \\
\hline & $\beta$ & $p$ & $\beta$ & $p$ & $\beta$ & $p$ & $\beta$ & $p$ & $\beta$ & $p$ \\
\hline (Constante) & & 0 & & 0 & & 0.21 & & 0.87 & & 0.48 \\
\hline África & -0.06 & 0.09 & -0.08 & 0 & -0.10 & 0 & -0.09 & 0 & -0.09 & 0 \\
\hline Europa & 0.07 & 0.05 & 0.02 & 0.44 & 0.01 & 0.64 & 0.02 & 0.53 & 0.02 & 0.53 \\
\hline Latinoamérca & 0.13 & 0 & 0.02 & 0.41 & 0 & 0.95 & 0 & 0.85 & 0.01 & 0.76 \\
\hline Tenacidad & & & 0.48 & 0 & 0.41 & 0 & 0.36 & 0 & 0.28 & 0 \\
\hline Apoyo autóctonos & & & & & 0.24 & 0 & 0.18 & 0 & 0.17 & 0 \\
\hline Apoyo familia & & & & & & & 0.15 & 0 & 0.15 & 0 \\
\hline Control personal & & & & & & & & & 0.11 & 0 \\
\hline
\end{tabular}

Para poner a prueba la hipótesis 3, se analiza el efecto de la interacción entre región de procedencia y las cuatro variables predictoras significativas mediante el modelo 1 del macro PROCESS diseñado para SPSS (Hayes, 2013). Para comprobar la interacción con cada una de las cuatro variables, se proponen cuatro modelos, en los que se va calculando sucesivamente la interacción con cada una de los predictores, manteniendo constantes los otros tres, como variables de control. En el primer modelo, el cambio en $\mathrm{R}^{2}$ se debe a la ausencia de interacción $<0.0001(F=1.06, p=0.36)$, el origen del inmigrante no modula la relación positiva de la tenacidad con la satisfacción con la vida. En la Tabla 3 puede observarse, no obstante, que el efecto de la tenacidad sobre la satisfacción con la vida es menor en el caso de los europeos y mayor en los latinos. La segunda ecuación introduce la interacción con el control personal, que tampoco es estadísticamente significativa: $\mathrm{R}^{2}$ debido a la interacción $<0.0001(\mathrm{~F}=$ 1.16, $p=0.33)$. La Tabla 3 muestra que, en este caso, es en los asiáticos en los que el control personal incide menos en la satisfacción vital. La tercera ecuación calcula la interacción con el apoyo social de los amigos inmigrantes; tampoco aquí hay interacción, siendo la $\mathrm{R}^{2}$ debido a la interacción $<0.0001(F=0.11$, $p=0.96) \mathrm{y}$, como se muestra en la tabla, no hay prácticamente diferencias del coeficiente en función del grupo de procedencia. Finalmente, se calcula la interacción con apoyo social de 
la familia, que no es significativa con una $\mathrm{R}^{2}$ debido a la interacción $<0.0001(F=1.14, p$ $=0.33)$. El peso del apoyo de la familia sobre la satisfacción con la vida es algo mayor en el caso de los latinoamericanos y los africanos. Por lo tanto, no se verifica la hipótesis 3 para ninguna de las variables predictoras de la satisfacción con la vida.

\section{TABLA 3}

Efecto condicional de los predictores sobre satisfacción con la vida según la región de origen

\begin{tabular}{|c|c|c|c|c|}
\hline Región de origen & Tenacidad & Control & Apoyo autóct & Apoyo familia \\
\hline África & $0.31 * *$ & $0.16^{* *}$ & $0.17 * *$ & $0.21 * *$ \\
\hline Europa & $0.21 * *$ & $0.10^{*}$ & $0.16^{* *}$ & $0.11^{*}$ \\
\hline Latinoamérica & $0.33^{* *}$ & $0.15^{* *}$ & $0.18^{* *}$ & $0.19 * *$ \\
\hline Asia & $0.27 * *$ & 0.04 & $0.20^{* *}$ & $0.12 *$ \\
\hline
\end{tabular}

Fuente: elaboración propia.

Se procede del mismo modo con la variable problemas mentales, cuyos resultados se resumen en la Tabla 4. En el primer paso se puede observar que no hay diferencias entre los cuatro grupos en problemas mentales. Mínimamente los europeos son los que puntúan más alto en esta variable $(M$ $=0.98)$, seguidos por los africanos $(M=0.96)$, los asiáticos $(\mathrm{M}=0.93)$ y los latinoamericanos $(M=0.91)$. En el segundo paso, la inclusión de la tenacidad aumenta la proporción de varianza explicada de problemas mentales. El paso tres también aumenta la $\mathrm{R}^{2}$ con la inclusión en la ecuación del apoyo social proporcionado por la familia. Finalmente, entra el control personal. Puede observarse que la mayor tenacidad y control personal, así como la recepción del apoyo social de la familia, se corresponden con menos problemas mentales. También se observa que la mínima diferencia inicial de los inmigrantes europeos tiene un incremento estadísticamente significativo a partir del paso dos y se mantiene hasta el final. Estos resultados no confirman la hipótesis uno y parcialmente la dos.

Como el caso anterior, hay que señalar la correlación alta entre las dos variables de resiliencia que se han retenido en la ecuación de regresión; sin que se hayan resentido excesivamente los diagnósticos de colinealidad. Así para la variable tenacidad, los índices son:
Tolerancia $=0.44 ; \mathrm{FIV}=2.23$. Para control personal: Tolerancia $=0.49 ; \mathrm{FIV}=2.02$. Solo cuando en la ecuación se introduce la variable control personal, aumentan moderadamente los problemas de colinealidad (Autovalor $=0.008$; Índice de condición $=24.77)$. Ello indica una cierta redundancia de esta variable respecto a la de tenacidad.

\section{TABLA 4}

Regresión múltiple por pasos con problemas mentales como criterio y con región de origen (variables ficticias), resiliencia (tenacidad, control personal y competencia social) y apoyo social (familiar, amigos autóctonos y amigos inmigrantes) como predictores

\begin{tabular}{|c|c|c|c|c|c|c|c|c|}
\hline & \multicolumn{2}{|c|}{ Paso 1} & \multicolumn{2}{|c|}{ Paso 2} & \multicolumn{2}{|c|}{ Paso 3} & \multicolumn{2}{|c|}{ Paso 4} \\
\hline & $\Delta R^{2}=0$ & $F=1.47$ & $\Delta R^{2}=0.0$ & $F=23.46$ & $\Delta R^{2}=0.1$ & $F=26.55$ & $\Delta R^{2}=0.1$ & $F=25.02$ \\
\hline & $\beta$ & $p$ & $\beta$ & $p$ & $\beta$ & $p$ & $\beta$ & $p$ \\
\hline (Constante) & & 0 & & 0 & & 0 & & 0 \\
\hline África & 0.03 & 0.34 & 0.04 & 0.23 & 0.04 & 0.24 & 0.04 & 0.26 \\
\hline Europa & 0.06 & 0.11 & 0.08 & 0.02 & 0.08 & 0.02 & 0.08 & 0.02 \\
\hline Latinoamérica & 0 & 0.82 & 0.05 & 0.19 & 0.05 & 0.17 & 0.04 & 0.20 \\
\hline Tenacidad & & & -0.29 & 0 & -0.21 & 0 & -0.09 & 0.02 \\
\hline Apoyo familia & & & & & -0.19 & 0 & -0.19 & 0 \\
\hline Control personal & & & & & & & -0.16 & 0 \\
\hline
\end{tabular}

El efecto de la interacción entre región de procedencia y las tres variables predictoras significativas se analiza mediante el modelo 1 del macro PROCESS, diseñado para SPSS (Hayes, 2013). Para comprobar la interacción con cada una de las tres variables se proponen tres modelos, en los que se va calculando sucesivamente la interacción con cada una de los predictores, manteniendo constantes los otros dos como covariables. En el primer modelo, el cambio en $\mathrm{R}^{2}$ se debe a la ausencia de interacción $<0.0001(F=0.48, p=0.7)$. El origen del inmigrante no modula la relación de la tenacidad con los problemas mentales. En la Tabla 5 puede observarse, no obstante, que el efecto negativo de la tenacidad sobre los problemas mentales es menor en el caso de los europeos y los asiáticos. La segunda ecuación introduce la interacción con el control personal, que tampoco es estadísticamente significativa; $\mathrm{R}^{2}$ debido a la interacción $<0.0001(F=0.09, p=0.96)$. Se ve en la tabla 5 que los coeficientes de los cuatro grupos apenas difieren. Finalmente, se calcula la interacción con apoyo social de la 
familia. En este caso la interacción es significativa con una $\mathrm{R}^{2}$ debido a la interacción $=0.01(\mathrm{~F}=$ $3.59, p=0.01)$. El peso del apoyo de la familia sobre la disminución de los problemas mentales es mayor en el caso de los latinoamericanos, seguidos por los asiáticos, siendo muy pequeño para los europeos y los africanos. Se confirma la hipótesis 3 para la influencia del apoyo de la familia sobre los problemas mentales.

\section{TABLA 5}

Efecto condicional de los predictores sobre problemas mentales según la región de origen

\begin{tabular}{lccc}
\hline $\begin{array}{l}\text { Región de } \\
\text { origen }\end{array}$ & Tenacidad & $\begin{array}{c}\text { Apoyo } \\
\text { familia }\end{array}$ & $\begin{array}{c}\text { Control } \\
\text { personal }\end{array}$ \\
\hline África & $-0.06 *$ & $-0.08 * *$ & $-0.06 *$ \\
Europa & -0.02 & $-0.07 * *$ & -0.03 \\
Latinoamérica & -0.05 & $-0.07 * *$ & $-0.14 * *$ \\
Asia & -0.03 & $-0.07 *$ & $-0.1 * *$ \\
\hline \multirow{4}{*}{$* *<<0.01$} & \\
& $* \mathrm{p}<0.05$
\end{tabular}

\section{Discusión y conclusiones}

Esta investigación ha analizado cómo se relacionan el apoyo social provisto por la familia, los amigos autóctonos e inmigrantes y la resiliencia, con la satisfacción vital y la salud mental en una amplia muestra de inmigrantes de distinta procedencia (África, Asia, Latinoamérica y Europa del Este).

En general, los resultados han confirmado parcialmente las hipótesis. Para la hipótesis 1, en la que pronosticamos encontrar diferencias en la satisfacción con la vida y los problemas mentales según la región de origen, se ha encontrado para la satisfacción vital que los latinoamericanos son los que muestran más satisfacción vital, mientras que los inmigrantes africanos son los que indican menor satisfacción con la vida. Estos resultados apoyan estudios previos con resultados similares (Moreno \& Hidalgo, 2011).

Respecto a los problemas de salud mental, no se han obtenido resultados significativos entre los inmigrantes de diferente procedencia. Sin embargo, estudios con inmigrantes asiáticos revelan que este colectivo es menos propenso a buscar ayuda psicológica ante problemas de ansiedad y estrés (Llop-Gironés, Vargas Lorenzo, Garcia-Subirats, Aller, \& Vázquez, 2014), por lo que quizá futuras investigaciones podrían dirigirse hacia el tipo de servicio de salud al que acude cada grupo de inmigrantes según su procedencia. Además, se ha encontrado que una de las barreras más notables a la hora de buscar o no la ayuda, junto con el idioma, es la barrera cultural. Algunos estudios han mostrado que los inmigrantes asiáticos de segunda generación obtienen puntuaciones similares a la población autóctona en cuanto al uso del servicio de salud mental (Abe-Kim et al., 2007). También se ha apuntado recientemente que el lugar de procedencia es un factor que determina una mejor o peor salud mental y una mayor o menor prevalencia de trastornos psiquiátricos en mayor medida que otros factores como la edad, el sexo o el estatus económico, con lo que deberíamos seguir atendiendo a dicho elemento diferenciador (Escobar, 1998).

Para la hipótesis 2, en la que se pronosticaba que las fuentes de apoyo y las dimensiones de la resiliencia se relacionaban con mayor satisfacción con la vida y menor incidencia de problemas mentales, se ha comprobado que la percepción de apoyo de las personas inmigrantes se relaciona positivamente con la satisfacción vital y la salud mental. De forma más específica, la percepción del apoyo familiar y de los amigos autóctonos e inmigrantes se relaciona positivamente con la valoración subjetiva de la vida, resultados que coinciden con los obtenidos por otros estudios (Herrero et al., 2012). En cambio, es la percepción del apoyo familiar la que se relaciona de forma más importante con una menor incidencia de los problemas mentales. Estos datos siguen la misma línea de otros que indican que el apoyo social se relaciona con menos problemas mentales y especialmente el apoyo que proporciona la familia (Runyan et al., 1998).

Sin embargo, el apoyo de los amigos inmigrantes no se relaciona con la satisfacción vital y la salud mental. Estos resultados coinciden con aquellos estudios que muestran cómo el apoyo de los amigos autóctonos les hace 
sentir más integrados en el país de acogida y percibir más satisfacción vital (Domínguez \& Hombrados, 2012). También estos resultados pueden explicarse desde el Modelo de Convoy social (Khan \& Antonucci, 1980), que presenta las necesidades de apoyo de los inmigrantes desde un plano dinámico. Las necesidades de apoyo van evolucionando conforme progresa el proceso migratorio. En un principio, cuando los inmigrantes llegan al nuevo país, buscan el contacto y el apoyo de otros compatriotas, pero conforme van asentándose e integrándose en el país de acogida, buscan el contacto y el apoyo de la población autóctona. En el estudio, el tiempo medio que llevan viviendo los inmigrantes en España es de 10 años, por lo que posiblemente este tiempo les ha permitido ir desarrollando lazos de apoyo e interacciones positivas con la población autóctona. Este aspecto deberá seguir analizándose en futuros estudios incluyendo la variable tiempo en un análisis longitudinal de la construcción de las redes de apoyo en el proceso migratorio.

En el presente estudio se ha comprobado que cuando se percibe apoyo de la familia, este es el mejor protector para la salud mental de los inmigrantes. Para la satisfacción vital, el apoyo familiar y el de amigos autóctonos son buenos predictores.

En cuanto a la resiliencia, los resultados indican que la capacidad resiliente de los inmigrantes se relaciona positivamente con la satisfacción vital y la salud mental. En concreto, las dimensiones de tenacidad y el control personal. En cambio, la competencia social no se relaciona. Una posible explicación es que en la escala empleada (Serrano-Parra et al., 2012), la competencia social se mide únicamente con dos ítems y quizás sería necesario incluir más indicadores para medir esta dimensión, por lo que en futuros estudios se tendrá que seguir indagando sobre este aspecto.

Básicamente, la hipótesis 3 no se confirma. No ha habido efectos de interacción entre las variables predictoras y la región de origen a la hora de explicar la satisfacción con la vida y los problemas mentales de los inmigrantes. La relación positiva que mantiene el apoyo social y la resiliencia con la satisfacción vital es de magnitud similar en los cuatro grupos de inmigrantes. Sucede otro tanto en lo que se refiere al criterio problemas mentales; no hay grandes diferencias entre los cuatro grupos a la hora de su explicación por la tenacidad y el control personal, pero sí en lo que se refiere a la influencia del apoyo de la familia. En este caso, son los latinos los que tienen menos problemas mentales en la medida en que reciben más apoyo social de sus familias.

Habría que seguir profundizando en este aspecto y analizar, como apuntan algunos estudios, que las dificultades de adaptación al país están condicionadas según la mayor o menor diferencia entre los códigos culturales, las normas y los valores del país extranjero (Zlobina, Basabe, \& Páez, 2004). Otros estudios muestran además, que los asiáticos son menos propensos que los inmigrantes europeos a buscar apoyo para afrontar el estrés y las situaciones de vulnerabilidad a las que se ven expuestos en su proceso de emigración, manifestando su preocupación por perder la aceptación de los otros miembros del grupo (Taylor et al., 2004).

La población latinoamericana en general ha obtenido mejores índices en las variables analizadas. Posiblemente es el grupo cultural más cercano ya que, como la literatura manifiesta, el idioma común aporta facilidades para el ajuste del inmigrante en el nuevo país, tanto en sus primeros momentos como en su proceso de integración (Arenas \& Alfonso, 2016). Así, los latinoamericanos se han convertido en el grupo mejor valorado por el colectivo autóctono (García, Jiménez, \& Redondo, 2009), según algunos autores porque colaboran con la sociedad y aportan beneficios sin los costes ni dificultades culturales y educativas que conlleva el inmigrar a un país con una lengua y cultura ajena (Roth, 2007). Este hecho nos hace revalidar la importancia de fomentar la percepción de apoyo y las interacciones positivas entre la población autóctona y la inmigrante.

El estudio se ha realizado con una gran muestra de inmigrantes por lo que creemos que estos resultados tienen una gran aplicabilidad e interés psicosocial. Conocer los aspectos que influyen en la adaptación a un nuevo país, así como tener 
en cuenta que la inmigración es un fenómeno complejo que no puede generalizarse, sino que debe ser tratado con singularidad, puede prevenir las dificultades en que se ven inmersas las personas inmigrantes en España, así como luchar contra el choque cultural y sus efectos negativos.

Las posibles vías de intervención deberían dirigirse al desarrollo y potenciación de la capacidad resiliente, para que puedan adquirir las competencias necesarias que les permitan afrontar la pluralidad de situaciones estresantes y adversas a las que se ven enfrentados. Además, se propone el desarrollo de pautas de interacción positiva entre autóctonos e inmigrantes, para promover la percepción positiva de apoyo entre ambos y prestar especial atención a aquellos inmigrantes que carecen de apoyo familiar (e.g. dejar la familia en el lugar de origen).

Este estudio no está exento de limitaciones; se trata de una investigación transversal, por lo que sería interesante analizar todas las variables a lo largo del tiempo para comprobar cómo se desarrolla la resiliencia y cómo se van creando las redes sociales. Sería interesante incluir más variables de estudio como apuntan algunos modelos (Khan \& Antonucci, 1980), y analizar las diferencias entre los grupos de inmigrantes atendiendo a sus características sociodemográficas. También futuros estudios deberán identificar en cada grupo de inmigrantes aquellos elementos que favorecen la percepción de apoyo y la resiliencia, atendiendo a las diferentes identidades culturales. Conocer estos aspectos diferenciales es fundamental para establecer estrategias específicas de intervención atendiendo a las necesidades de los diferentes grupos de inmigrantes.

\section{Agradecimientos}

Este estudio ha estado financiado por el proyecto I + D con referencia PSI2013-40508-P.

Nuestro agradecimiento a todas las personas inmigrantes que desinteresadamente participaron en nuestro estudio.

\section{Referencias}

Abe-Kim, J., Takeuchi, D. T., Hong, S., Zane, N., Sue, S., Spencer, M. S., Appel, H., Nicdao, E., \& Alegría, M. (2007). Use of mental health-related services among immigrant and US-born Asian Americans: Results from the National Latino and Asian American study. American journal of public health, 97(1), 91-98. https:// doi.org/10.2105/AJPH.2006.098541

Arenas, P., \& Alfonso, U. (2016). Estrategias de aculturación e identidad étnica: un estudio en migrantes sur-sur en el norte de Chile. Universitas Psychologica, 15(1). https:// doi.org/10.11144/Javeriana.upsy15-1.eaie

Ayyash-Abdo, H., \& Alamuddin, R. (2007). Predictors of subjective well-being among college youth in Lebanon. The Journal of Social Psychology, 147(3), 265-284. https:// doi.org/10.3200/SOCP.147.3.265-284

Barudy, J., \& Dantagnan, M. (2005). Los buenos tratos a la infancia: Parentalidad, apego y resiliencia. Barcelona: Gedisa.

Checa, J. C., \& Monserrat, M. (2015). La integración social de los hijos de inmigrantes africanos, europeos del este y latinoamericanos: un estudio de caso en España. Universitas Psychologica, 14(2), 475-486. https://doi.org/10.11144/ Javeriana.upsy14-2.lish

Connor, K. M., \& Davidson, J. R. (2003). Development of a new resilience scale: The Connor-Davidson resilience scale (CD. RISC). Depression and anxiety, 18(2), 76-82. https://doi.org/10.1002/da.10113

Diener, E. (2006). Guidelines for national indicators of subjective well-being and ill-being. Journal of Happiness Studies, 7, 397-404. https://doi.org/10.1007/ s10902-006-9000

Domínguez, J. M., \& Hombrados, M. I. (2012). Social support and happiness in immigrant women in Spain. Psychological Reports, 110(3), 977-990. https:// doi.org/10.2466/17.02.20.21.PR0.110.3.977-990

Escobar, J. I. (1998). Immigration and mental health: Why are immigrants 
better off? Archives of General Psychiatry, 55(9), 781-782. https://doi.org/10.1001/ archpsyc.55.9.781

Gagnon, A. J., \& Stewart, D. E. (2014). Resilience in international migrant women following violence associated with pregnancy. Archives of women's mental health, 17(4), 303-310. https:// doi.org/10.1007/s00737-013-0392-5.

García, A., Jiménez, B., \& Redondo, A. (2009). La inmigración latinoamericana en España en el siglo XXI. Investigaciones geográficas, $70,55-70$.

García, M. A., Hombrados-Mendieta, I., \& Gómez-Jacinto, L. (2016). A Multidimensional Approach to Social Support: The Questionnaire on the Frequency of and Satisfaction with Social Support (QFSSS). Anales de Psicología, 32 (2), 501-515. https://doi.org/10.6018/ analesps.32.2.201941

Hayes, A. F. (2013). Introduction to mediation, moderation, and conditional process analysis: A regression-bases approach. New York: The Guilford Press.

Hernández, S., Alonso, E., \& Pozo, C. (2006). Social support interventions in migrant populations. British Journal of Social Work, 36(7), 1151-1169. https://doi.org/10.1093/ bjsw/bch396

Herrero, J., \& Gracia, E. (2011). Covariates of Subjective well-being among Latin American immigrants in Spain: the role of social integration in the community. Journal of Community Psychology, 39(7), 761-775. https://doi.org/10.1002/jcop.20468

Herrero, J., Gracia, E., Fuente, A., \& Lila, M. (2012). Desorden social, integración social y bienestar subjetivo en inmigrantes latinoamericanos en España. Anales de psicología, 28(2), 505-514. https:// doi.org/10.6018/analesps.28.2.148721

Herrman, H., Stewart, D. E., Diaz-Granados, N., Berger. E. L., Jackson, B., \& Yuen, T. (2011). What is resilience? Can J Psychiatry 56(5), 258. https:// doi.org/10.1177/070674371105600504
Hombrados-Mendieta, I., Gómez-Jacinto, L., Domínguez-Fuentes, J. M., \& GarcíaLeiva, P. (2013). Sense of community and satisfaction with life among immigrants and the native population. Journal of Community Psychology, 41(5), 601-614. https://doi.org/10.1002/jcop.21559

Hovey, J. D., \& Magana, C. G. (2002). Psychosocial predictors of anxiety among immigrant Mexican migrant farmworkers. Implications for prevention and treatment. Cultural Diversity and Ethnic Minority Psychology, 8(3), 274-289. https:// doi.org/10.1037/1099-9809.8.3.274

Jasinskaja-Lahti, I., Liebkind, K., Jaakkola, M., \& Reuter, A. (2006). Perceived discrimination, social support networks, and psychological well-being among three immigrant groups. Journal of CrossCultural Psychology, 37(3), 293-311. https:// doi.org/10.1177/0022022106286925

Khan, R. L., \& Antonucci, T. C. (1980). Convoys over the life course: attachment, roles and social support. In P. Baltes \& O. Brim (Eds.), Life span development and behavior, 3 , 253-286. San Diego CA: Academic Press.

Kiang, L., Grzywacz, J., Marín, A., Arcury, T., \& Quandt, S. (2010). Mentalhealth in immigrants from nontraditional receiving sites. Cultural Diversity and Ethnic Minority Psychology, 16(3), 386-394. https:// doi.org/10.1037/a0019907

Lai, D. W., \& McDonald, R. (1995). Life satisfaction of Chinese elderly immigrants in Calgary. Canadian Journal of Aging, 14(3), 536-552. https://doi.org/10.1017/ S0714980800009107

Lin, N., Dean, A., \& Ensel, W. M. (1986). Social support, life events and depression. Nueva York: Academic Press.

Llop-Gironés, A., Vargas Lorenzo, I., Garcia-Subirats, I., Aller, M. B., \& Vázquez Navarrete, M. L. (2014). Acceso a los servicios de salud de la población inmigrante en España. Revista Española de Salud Pública, 88(6), 715-734. https://doi.org/10.4321/ S1135-57272014000600005 
Luthar, S.S., \& Cichetti, D. (2000). The construct of resilience: Implications for interventions and social policies. Development and Psychopathology, 12, 857-85. https://doi.org/10.1017/ S0954579400004156

Luthar, S. S., Cicchetti, D., \& Becker, B. (2000). The construct of resilience: A critical evaluation and guidelines for future work. Child development, 71(3), 543-562. https:// doi.org/10.1111/1467-8624.00164

Mahendra, V., \& Agarwal, G. (2016). Quality of life, psychological impairment, and resilience among employment-restricted immigrants. Indian Journal of Health $\mathbb{E}$ Wellbeing, 7(11), 1042-1047.

Malone, M. E., \& Dooley, J. P. (2006). 'DWELLING IN DISPLACEMENT' Meanings of 'community' and sense of community for two generations of Irish people living in NorthWest London. Community, work and family, 9(1), 11-28. https:// doi.org/10.1080/13668800500420947

Moreno, M. P., \& Hidalgo, M. C. (2011). Medición y predicción de la satisfacción con la vida en inmigrantes empleados en España. Diferencias según su situación administrativa. Anales de psicología, 27(1), 179-185.

Newman, R. (2005). APA's resilience initiative. Professional Psychology-Research and Practice, 36(3), 227. https:// doi.org/10.1037/0735-7028.36.3.227

Obrist, B., \& Büchi, S. (2008). Stress as an idiom for resilience: health and migration among sub- Saharan Africans in Switzerland. Anthropology Eु medicine, 15(3), 251-261. https:// doi.org/10.1080/13648470802357596.

Panchang, S., Dowdy, H., Kimbro, R., \& Gorman, B. (2016). Self-rated health, gender, and acculturative stress among immigrants in the US: New roles for social support. International Journal of Intercultural Relations, 55, 120-132. https:// doi.org/10.1016/j.ijintrel.2016.10.0
Patiño, C., \& Kirchner, T. (2008): Estrés y coping en inmigrantes latinoamericanos residentes en Barcelona. Revista Iberoamericana de psicología: Ciencia y tecnología, 1(1), 29-38.

Pavot, W., \& Diener, E. (1993). Review of the satisfaction With life scale. Psychological assessment, $\quad 5(2), \quad$ 164-170. https:// doi.org/10.1007/978-90-481-2354-45

Pereda, E., \& Pereira, R. (2006). Resiliencia e inmigración. Tercer Curso de Terapéutica Familiar. Escuela Vasco-Navarra de Terapia Familiar.

Roth, J. O. (2007). Lengua y migraciones: aspectos culturales de la inmigración latinoamericana en España. Análisis del Real Instituto Elcano (ARI), 36, 1.

Rudmin, F. (2009). Constructs, measurements and models of acculturation and acculturative stress. International Journal of Intercultural Relations, 33, 106-123. https:// doi.org/10.1016/j.ijintrel.2008.12.001

Runyan, D. K., Hunter, W. M., Socolar, R. R., Amaya-Jackson, L., English, D., Landsverk, J., \& Mathew, R. M. (1998). Children who prosper in unfavorable environments: the relationship to social capital. Pediatrics, 101(1), 12-18. https:// doi.org/10.1542/peds.101.1.12

Rutter, M. (1993). Resilience: Some conceptual considerations. Journal of Adolescent Health, 14(8), 626-631. New York: Society for Adolescent Medicine. https:// doi.org/10.1016/1054-139X(93)90196-V

Safi, M. (2010). Immigrants' life satisfaction in Europe: Between assimilation and discrimination. European Sociological Review, 26(2), 159-176. https:// doi.org/10.1093/esr/jcp013

Serrano-Parra, M.D., Garrido-Abejara, M., Notario-Pacheco, B., Bartolomé-Gutierrez, R., Solera- Martínez, M., \& MartínezVizcaino, V. (2012). Validity of the Connor Davidson Resilience scale (CD. RISC) in people from 60-to-75 years old. International Journal of Psychological Research, 5(2), 49-57.

Shavitt, S., Cho, Y. I., Johnson, T. P., Jiang, D., Holbrook, A., \& 
Stavrakantonaki, M. (2016). Culture moderates the relation between perceived stress, social support, and mental and physical health. Journal of CrossCultural Psychology, 47(7), 956-980. https:// doi.org/10.1177/0022022116656132

Shoval, G., Schoen, G., Vardi, N., \& Zalsman, G. (2007). Suicide in Ethiopian immigrants in Israel: a case for study of the geneticenvironmental relation in suicide. Archives of Suicide Research, 11(3), 247-253. https:// doi.org/10.1080/13811110701402603

Taylor, S. E., Sherman, D. K., Kim, H. S., Jarcho, J., Takagi, K., \& Dunagan, M. S. (2004). Culture and social support: who seeks it and why? Journal of personality and social psychology, 87(3), 354. https:// doi.org/10.1037/0022-3514.87.3.354

Tormo, M., Salmerón, D., Colorado-Yohar, S., Ballesta, M., Dios, S., Martínez- Fernández, C., Pérez- Flores, D., García-Pérez, V., Palomar, J., Torres, A., \& Navarro, C. (2015). Resultados de dos encuestas dirigidas a inmigrantes y nativos del sureste español: salud, uso de servicios y necesidad de asistencia médica. Salud pública de México, 57(1), 38-49.

Tunstall, H., Shortt, N. K., Pearce, J. R., \& Mitchell, R. J. (2015). Difficult Life Events, Selective Migration and Spatial Inequalities in Mental Health in the UK. PLoS ONE, 10(5), e0126567. https://doi.org/10.1371/ journal.pone. 0126567

Ugalde-Watson, K., Smith-Castro, V., MorenoSalas, M., \& Rodríguez-García, J. M. (2011). Estructura, correlatos y predictores del estrés por aculturación. El caso de personas refugiadas colombianas en Costa Rica. Universitas Psychologica, 10(3), 759.

Vélez, M. G., Aguado, O. V., \& Pérez, P. Á. (2013). El análisis de la resiliencia en personas que constituyen parejas mixtas en Andalucía/Analysis of Resilience in Members of Mixed Couples in Andalusia. Cuadernos de Trabajo Social, 26(2), 275. https://doi.org/10.5209/ rev_CUTS.2013.v26.n2.41474
Villa, I., Zuluaga, C., \& Restrepo, L. (2013). Propiedades psicométricas del Cuestionario de Salud General de Goldberg GHQ-12 en una institución hospitalaria de la ciudad de Medellín. Avances en Psicología Latinoamericana/Bogotá, 31 (3), 532-545.

Wang, S. W., \& Lau, A. S. (2015). Mutual and non-mutual social support: cultural differences in the psychological, behavioral, and biological effects of support seeking. Journal of CrossCultural Psychology, 46(7), 916-929. https:// doi.org/10.1177/0022022115592967

Xu, L., \& Chi, I. (2013). Acculturative stress and depressive symptoms among Asian immigrants in the United States: The roles of social support and negative interaction. Asian American Journal of Psychology, 4(3), 217-226. https://doi.org/10.1037/a0030167

Zarza, M. J., \& Prados, M. I. S. (2007). Estre\#s de adaptacio\# $\mathrm{n}$ sociocultural en inmigrantes latinoamericanos residentes en Estados Unidos vs Espan\#a: una revisión bibliogra\#fica. Anales de Psicología, 23(1), 72-84.

Zlobina, A., Basabe, N., \& Páez, D. (2004). Adaptación de los inmigrantes extranjeros en España: superando el choque cultural. Migraciones, 15, 43-84.

\section{Notas}

* Artículo de investigación. 